\title{
88. A Remark concerning Probabilities on Inheritance in Consanguineous Families
}

\author{
By Yûsaku Komatu and Han NishimiYa \\ Department of Mathematics, Tokyo Institute of Technology \\ (Comm. by T. Furuhata, M.J.A., June 13, 1955)
}

In a series of papers ${ }^{1)}$ we have systematically derived formulas for probabilities of various combinations of consanguineous genotypes.

Having commenced with simple lineal combination, we first have shown that the reduced probability of mother- $n$th descendant combination is given $b^{2)}$

$$
\kappa_{n}(\alpha \beta ; \xi \eta)=\bar{A}_{\bar{\xi} \eta}+2^{-n+1} Q(\alpha \beta ; \xi \eta),
$$

and then that the reduced probability of parents- $n$th descendant combination is given by ${ }^{3)}$

$$
\varepsilon_{n}\left(\alpha \beta, \gamma \delta ; \xi_{\eta}\right)=\bar{A}_{\xi \eta}+2^{-n+1} E\left(\alpha \beta, \gamma \delta ; \xi_{\eta}\right) .
$$

The values of the quantities $Q$ and $E$ are also fully set out there.

Comparison of these listed tables shows now that there holds a very remarkable relation

$$
E\left(\alpha \beta, \gamma \delta ; \xi_{\eta}\right)=Q\left(\alpha \beta ; \xi_{\eta}\right)+Q\left(\gamma \delta ; \xi_{\eta}\right) .
$$

It is to be regretted that we have overlooked this important fact. If it had been perceived at that time, several relations concerning $E$ would have been much simply established.

Especially, the notation $C\left(\alpha \beta, \gamma \delta ; \xi_{\eta}\right)$ introduced $^{4)}$ for expressing the probability of ancestor-parent-descendant combination would then become superfluous, since it is dependent. In fact, we have

$$
\begin{aligned}
C\left(\alpha \beta, \gamma \delta ; \xi_{\eta}\right) & =2 \sum_{a \leq b} C_{0}(\alpha \beta, \gamma \delta ; a b) \kappa\left(a b ; \xi_{\eta}\right) \\
& =2 \sum_{a \leq b}^{\infty} C_{0}(\alpha \beta, \gamma \delta ; a b) Q\left(a b ; \xi_{\eta}\right) \\
& =4 \sum_{c \leq s ; a, a, s} Q(\alpha \beta ; c d) \varepsilon(c d, \gamma \delta ; a b) Q\left(a b ; \xi_{\eta}\right) \\
& =2 \sum_{c \leq d} Q(\alpha \beta ; c d) E\left(c d, \gamma \delta ; \xi_{\eta}\right) \\
& =2 \sum_{\delta \leq d} Q(\alpha \beta ; c d)\left\{Q\left(c d ; \xi_{\eta}\right)+Q\left(\gamma \delta ; \xi_{\eta}\right)\right\} \\
& =Q\left(\alpha \beta ; \xi_{\eta}\right) .
\end{aligned}
$$

Thus, for the probability of ancestors-descendant combination

1) Y. Komatu and H. Nishimiya, Probabilities on inheritance in consanguineous families. I-XIII. Proc. Japan Acad. 30 (1954), 42-45; 46-48; 49-52; 148-151; 152-155; $236-240$; 241-244; 245-247; 636-640；641-649；650-654; 31 (1955), 186-189; 190-194.

2) Cf. I, p. 43.

3) Cf. I, p. 44.

4) Cf. IV, p. 149. 
$\varepsilon_{\mu v ; n}(\alpha \beta, \gamma \delta ; \xi \eta)$, the distinction in the formulas according to $\mu=\nu=0$, $\mu>0=\nu$ (or $\mu=0<\nu$ ), and $\mu, \nu>0$ is really unnecessary provided $n>1$. Three formulas ${ }^{5)}$ may be unified, in general, into a single formula

$$
\varepsilon_{\mu \nu ; n}\left(\alpha \beta, \gamma \delta ; \xi_{\eta}\right)=\vec{A}_{\xi \eta}+2^{-n+1}\left\{2^{-\mu} Q\left(\alpha \beta ; \xi_{\eta}\right)+2^{-\nu} Q\left(\gamma \delta ; \xi_{\eta}\right)\right\}
$$

valid for any $\mu, \nu \geqq 0$ provided $n>1$.

Based on the identity just mentioned, several relations would then be accordingly more readily deducible.

5) Cf. I, p. 44; IV, p. 149; IV, p. 150. 